\title{
Fractional Crank-Nicolson-Galerkin Finite Element Methods for Nonlinear Time Fractional Parabolic Problems with Time Delay
}

\author{
Lili Li $\mathbb{D}^{1},{ }^{1}$ Mianfu She $\mathbb{D}{ }^{1}$ and Yuanling Niu $\mathbb{D}^{2}$ \\ ${ }^{1}$ School of Mathematics and Statistics, Huazhong University of Science and Technology, Wuhan 430074, China \\ ${ }^{2}$ School of Mathematics and Statistics, Central South University, Changsha 410083, China \\ Correspondence should be addressed to Yuanling Niu; yuanlingniu@csu.edu.cn
}

Received 11 March 2021; Accepted 18 March 2021; Published 31 March 2021

Academic Editor: Qifeng Zhang

Copyright (C) $2021 \mathrm{Lili} \mathrm{Li} \mathrm{et} \mathrm{al.} \mathrm{This} \mathrm{is} \mathrm{an} \mathrm{open} \mathrm{access} \mathrm{article} \mathrm{distributed} \mathrm{under} \mathrm{the} \mathrm{Creative} \mathrm{Commons} \mathrm{Attribution} \mathrm{License,} \mathrm{which}$ permits unrestricted use, distribution, and reproduction in any medium, provided the original work is properly cited.

\begin{abstract}
A linearized numerical scheme is proposed to solve the nonlinear time-fractional parabolic problems with time delay. The scheme is based on the standard Galerkin finite element method in the spatial direction, the fractional Crank-Nicolson method, and extrapolation methods in the temporal direction. A novel discrete fractional Grönwall inequality is established. Thanks to the inequality, the error estimate of a fully discrete scheme is obtained. Several numerical examples are provided to verify the effectiveness of the fully discrete numerical method.
\end{abstract}

\section{Introduction}

In this paper, we consider the linearized fractional CrankNicolson-Galerkin finite element method for solving the nonlinear time-fractional parabolic problems with time delay

$$
\begin{cases}{ }^{R} D_{t}^{\alpha} u-\Delta u=f(t, u(x, t), u(x, t-\tau)), & \text { in } \Omega \times(0, T], \\ u(x, t)=\varphi(x, t), & \text { in } \Omega \times(-\tau, 0], \\ u(x, t)=0, & \text { on } \partial \Omega \times(0, T],\end{cases}
$$

where $\Omega$ is a bounded convex and convex polygon in $R^{2}$ (or polyhedron in $R^{3}$ ) and $\tau$ is the delay term. ${ }^{R} D_{t}^{\alpha} u$ denotes the Riemann-Liouville fractional derivative, defined by

$$
{ }^{R} D_{t}^{\alpha} u(\cdot, t)=\frac{1}{\Gamma(1-\alpha)} \frac{\partial}{\partial t} \int_{0}^{t}(t-s)^{-\alpha} u(\cdot, s) d s, \quad 0<\alpha<1 .
$$

The nonlinear fractional parabolic problems with time delay have attracted significant attention because of their wide range of applications in various fields, such as biology, physics, and engineering [1-9]. Recently, plenty of numerical methods were presented for solving the linear time-fractional diffusion equations. For instance, Chen et al. [10] used finite difference methods and the Kansa method to approximate time and space derivatives, respectively. Dehghan et al. [11] presented a fully discrete scheme based on the finite difference methods in time direction and the meshless Galerkin method in space direction and proved the scheme was unconditionally stable and convergent. Murio [12] and Zhuang and Liu [13] proposed a fully implicit finite difference numerical scheme and obtained unconditionally stability. Jin et al. [14] derived the time-fractional Crank-Nicolson scheme to approximate Riemann-Liouville fractional derivative. Li et al. [15] used a transformation to develop some new schemes for solving the time-fractional problems. The new schemes admit some advantages for both capturing the initial layer and solving the models with small parameter $\alpha$. More studies can be found in [16-32].

Recently, it has been one of the hot spots in the investigations of different numerical methods for the nonlinear timefractional problems. For the analysis of the L1-type methods, we refer readers to the paper [33-40]. For the analysis of the convolution quadrature methods or the fractional CrankNicolson scheme, we refer to the recent papers [41-46]. The key role in the convergence analysis of the schemes is the fractional Grönwall-type inequations. However, as 
pointed out in [47-49], the similar fractional Grönwall-type inequations can not be directly applied to study the convergence of numerical schemes for the nonlinear timefractional problems with delay.

In this paper, we present a linearized numerical scheme for solving the nonlinear fractional parabolic problems with time delay. The time Riemann-Liouville fractional derivative is approximated by the fractional Crank-Nicolson-type timestepping scheme, the spatial derivative is approximated by using the standard Galerkin finite element method, and the nonlinear term is approximated by the extrapolation method. To study the numerical behavior of the fully discrete scheme, we construct a novel discrete fractional type Grönwall inequality. With the inequality, we consider the convergence of the numerical methods for the nonlinear fractional parabolic problems with time delay.

The rest of this article is organized as follows. In Section 2 , we present a linearized numerical scheme for the nonlinear time-fractional parabolic problems with delay and main convergence results. In Section 3, we present a detailed proof of the main results. In Section 4, numerical examples are given to confirm the theoretical results. Finally, the conclusions are presented in Section 5.

\section{Fractional Crank-Nicolson-Galerkin FEMs}

Denote $\mathscr{T}_{h}$ is a shape regular, quasiuniform triangulation of the $\Omega$ into $d$-simplexes. Let $h=\max _{K \varepsilon \mathscr{T}_{h}}\{\operatorname{diam} K\}$. Let $X_{h}$ be the finite-dimensional subspace of $H_{0}^{1}(\Omega)$ consisting of continuous piecewise function on $\mathscr{T}_{h}$. Let $\Delta t=\tau / m_{\tau}$ be the time step size, where $m_{\tau}$ is a positive integer. Denote $N=\lceil T / \Delta t\rceil$, $t_{j}=j \Delta t, j=-m_{\tau},-m_{\tau}+1, \cdots, 0,1,2, \cdots, N$.

The approximation to the Riemann-Liouville fractional derivative at point $t=t_{n-(\alpha / 2)}$ is given by [14]

${ }^{R} D_{t_{n-(\alpha / 2)}^{\alpha}}^{\alpha} u(x, t)=\Delta t^{-\alpha} \sum_{i=0}^{n} \omega_{n-i}^{(\alpha)} u\left(x, t_{i}\right)+\mathcal{O}\left(\Delta t^{2}\right):={ }^{R} D_{t_{\Delta t}}^{\alpha} u^{n}+\mathcal{O}\left(\Delta t^{2}\right)$,

where

$$
\omega_{i}^{(\alpha)}=(-1)^{i} \frac{\Gamma(\alpha+1)}{\Gamma(i+1) \Gamma(\alpha-i+1)} .
$$

For simplicity, denote $\|v\|=\left(\int_{\Omega}|v(x)|^{2} d x\right)^{1 / 2}, \eta^{n, \alpha}=(1-$ $(\alpha / 2)) \eta^{n}+(\alpha / 2) \eta^{n-1}, \quad \eta \wedge^{n, \alpha}=(2-(\alpha / 2)) \eta^{n-1}-(1-(\alpha / 2))$ $\eta^{n-2}, t_{n}^{\alpha}=(n \Delta t)^{\alpha}$.

With the notation, the fully discrete scheme is to find $U_{h}^{n} \varepsilon X_{h}$ such that

$$
\begin{aligned}
& \left\langle{ }^{R} D_{\Delta t}^{\alpha} U_{h}^{n}, v\right\rangle+\left\langle\nabla U_{h}^{n, \alpha}, \nabla v\right\rangle \\
& \quad=\left\langle f\left(t_{n-(\alpha / 2)}, \widehat{U}_{h}^{n, \alpha}, U_{h}^{n-m_{\tau}, \alpha}\right), v\right\rangle, \quad \forall v \mathcal{E} X_{h}, n=1,2, \cdots, N,
\end{aligned}
$$

and the initial condition

$$
U_{h}^{n}=R_{h} \varphi\left(x, t_{n}\right), \quad n=-m_{\tau},-m_{\tau}+1, \cdots, 0,
$$

where $R_{h}: H_{0}^{1}(\Omega) \rightarrow X_{h}$ is Ritz projection operator which satisfies the following equality [50]

$$
\left\langle\nabla R_{h} u, \nabla v\right\rangle=\langle\nabla u, \nabla v\rangle, \quad \forall u \varepsilon H_{0}^{1}(\Omega) \cap H^{2}(\Omega), v \varepsilon X_{h} .
$$

We present the main convergence results here and leave their proof in the next section.

Theorem 1. Suppose the system (1) has a unique solution $u$ satisfying

$$
\begin{gathered}
\left\|u_{0}\right\|_{H^{r+1}}+\|u\|_{C\left([0, T] ; H^{r+1}\right)}+\left\|u_{t}\right\|_{C\left([0, T] ; H^{r+1}\right)}+\left\|u_{t t}\right\|_{C\left([0, T] ; H^{2}\right)} \\
+\left\|^{R} D_{\Delta t}^{\alpha} u\right\|_{C\left([0, T] ; H^{r+1}\right)} \leq K
\end{gathered}
$$

and the source term $f(t, u(x, t), u(x, t-\tau))$ satisfies the Lipschitz condition

$$
\begin{aligned}
& |f(t, u(x, t), u(x, t-\tau))-f(t, v(x, t), v(x, t-\tau))| \\
& \quad \leq L_{1}|u(x, t)-v(x, t)|+L_{2}|u(x, t, \tau)-v(x, t, \tau)|,
\end{aligned}
$$

where $K$ is a constant independent of $n, h$, and $\Delta t$ and $L_{1}$ and $L_{2}$ are given positive constants. Then, there exists a positive constant $\Delta t^{*}$ such that for $\Delta t \leq \Delta t^{*}$, the following estimate holds that

$$
\left\|u^{n}-U_{h}^{n}\right\| \leq C_{1}^{*}\left(\Delta t^{2}+h^{r+1}\right), \quad n=1,2, \cdots, N
$$

where $C_{1}^{*}$ is a positive constant independent of $h$ and $\Delta t$.

Remark 2. The main contribution of the present study is that we obtain a discrete fractional Grönwall's inequality. Thanks to the inequality, the convergence of the fully discrete scheme for the nonlinear time-fractional parabolic problems with delay can be obtained.

Remark 3. At present, the convergence of the proposed scheme is proved without considering the weak singularity of the solutions. In fact, if the initial layer of the problem is taken into account, there are some corrected terms at the beginning. Then, the scheme can be of order two in the temporal direction for nonsmooth initial data and some incompatible source terms. However, we still have the difficulties to get the similar discrete fractional Grönwall's inequality. We hope to leave the challenging problems in the future.

\section{Proof of the Main Results}

In this section, we will present a detailed proof of the main result. 
3.1. Preliminaries and Discrete Fractional Grönwall Inequality. Firstly, we review the definition of weights $\omega_{i}^{(\alpha)}$ and denote $g_{n}^{(\alpha)}=\sum_{i=0}^{n} \omega_{i}^{(\alpha)}$. Then, we can get

$$
\left\{\begin{array}{l}
\omega_{0}^{(\alpha)}=g_{0}^{(\alpha)}, \\
\omega_{i}^{(\alpha)}=g_{i}^{(\alpha)}-g_{i-1}^{(\alpha)}, \quad 1 \leq i \leq n .
\end{array}\right.
$$

Actually, it has been shown [51] that $\omega_{i}^{(\alpha)}$ and $g_{n}^{(\alpha)}$ process following properties:

(1) The weights $\omega_{i}^{(\alpha)}$ can be evaluated recursively, $\omega_{i}^{(\alpha)}$ $=(1-((\alpha+1) / i)) \omega_{i-1}^{(\alpha)}, i \geq 1, \omega_{0}^{(\alpha)}=1$

(2) The sequence $\left\{\omega_{i}^{(\alpha)}\right\}_{i=0}^{\infty}$ are monotone increasing -1 $<\omega_{i}^{(\alpha)}<\omega_{i+1}^{(\alpha)}<0, i \geq 0$

(3) The sequence $\left\{g_{i}^{(\alpha)}\right\}_{i=0}^{\infty}$ are monotone decreasing, $g_{i}^{(\alpha)}>g_{i+1}^{(\alpha)}$ for $i \geq 0$ and $g_{0}^{(\alpha)}=1$

Noticing the definition of $g_{i}^{(\alpha)},{ }^{R} D_{\Delta t}^{\alpha} u^{n}$ can be rewritten as

$$
{ }^{R} D_{\Delta t}^{\alpha} u^{n}=\Delta t^{-\alpha} \sum_{i=1}^{n}\left(g_{i}^{(\alpha)}-g_{i-1}^{(\alpha)}\right) u^{n-i}+\Delta t^{-\alpha} g_{0}^{(\alpha)} u^{n}
$$

In fact, rearranging this identity yields

$$
{ }^{R} D_{\Delta t}^{\alpha} u^{n}=\Delta t^{-\alpha} \sum_{i=1}^{n} g_{n-i}^{(\alpha)} \delta_{t} u^{i}+\Delta t^{-\alpha} g_{n}^{(\alpha)} u^{0},
$$

where $\delta_{t} u^{i}=u^{i}-u^{i-1}$.

Lemma 4 (see [51]). Consider the sequence $\left\{\phi_{n}\right\}$ given by

$$
\phi_{0}=1, \phi_{n}=\sum_{i=1}^{n}\left(g_{i-1}^{(\alpha)}-g_{i}^{(\alpha)}\right) \phi_{n-i}, \quad n \geq 1 .
$$

Then, $\left\{\phi_{n}\right\}$ satisfies the following properties:

(i) $0<\phi_{n}<1, \sum_{i=j}^{n} \phi_{n-i} g_{i-j}^{(\alpha)}=1,1 \leq j \leq n$

(ii) $1 /(\Gamma(\alpha)) \sum_{i=1}^{n} \phi_{n-i} \leq n^{\alpha} / \Gamma(1+\alpha)$

(iii) $1 /(\Gamma(\alpha) \Gamma(1+(k-1) \alpha)) \sum_{i=1}^{n-1} \phi_{n-i} i^{(k-1) \alpha} \leq n^{k \alpha} / \Gamma(1+$ $\alpha), k=1,2 \cdots$
Lemma 5 (see [51]). Consider the matrix

$$
W=2 \mu(\Delta t)^{\alpha}\left(\begin{array}{ccccc}
0 & \phi_{1} & \cdots & \phi_{n-2} & \phi_{n-1} \\
0 & 0 & \cdots & \phi_{n-3} & \phi_{n-2} \\
\vdots & \vdots & \ddots & \vdots & \vdots \\
0 & 0 & \cdots & 0 & \phi_{1} \\
0 & 0 & \cdots & 0 & 0
\end{array}\right)_{n \times n}
$$

Then, $W$ satisfies the following properties:

(i) $W^{l}=0, l \geq n$

(ii) $W^{k \overleftarrow{e}} \leq(1 /(\Gamma(1+k \alpha)))\left[\left(2 \Gamma(\alpha) \mu t_{n}^{\alpha}\right)^{k},\left(2 \Gamma(\alpha) \mu t_{n-1}^{\alpha}\right)^{k}\right.$, $\left.\cdots,\left(2 \Gamma(\alpha) \mu t_{1}^{\alpha}\right)^{k}\right]^{\prime}, k=0,1,2, \cdots$

(iii) $\sum_{k=0}^{l} W^{k \overleftarrow{e}}=\sum_{k=0}^{n-1} W^{k \overleftarrow{e}} \leq\left[E_{\alpha}\left(2 \Gamma(\alpha) \mu t_{n}^{\alpha}\right), E_{\alpha}(2 \Gamma(\alpha)\right.$ $\left.\left.\mu t_{n-1}^{\alpha}\right), \cdots, E_{\alpha}\left(2 \Gamma(\alpha) \mu t_{1}^{\alpha}\right)\right]^{\prime}, l \geq n$

where $\overleftarrow{e}=[1,1, \cdots, 1]^{\prime} \in \mathbb{R}^{n}, \mu$ is a constant

Theorem 6. Assuming $\left\{u^{n} \mid n=-m,-m+1, \cdots, 0,1,2, \cdots\right\}$ and $\left\{f^{n} \mid n=0,1,2, \cdots\right\}$ are nonnegative sequence, for $\lambda_{i}>0$ , $i=1,2,3,4,5$, if

$$
{ }^{R} D_{\Delta t}^{\alpha} u^{j} \leq \lambda_{1} u^{j}+\lambda_{2} u^{j-1}+\lambda_{3} u^{j-2}+\lambda_{4} u^{j-m}+\lambda_{5} u^{j-m-1}+f^{j}, \quad j=1,2 \cdots,
$$

then, there exists a positive constant $\Delta t^{*}$, for $\Delta t<\Delta t^{*}$, the following holds

$$
\begin{aligned}
u^{n} \leq & 2\left(\lambda_{4} \frac{\Gamma(\alpha) t_{n}^{\alpha}}{\Gamma(1+\alpha)} M+\lambda_{5} \frac{\Gamma(\alpha) t_{n}^{\alpha}}{\Gamma(1+\alpha)} M+\max _{1 \leq j \leq n} f^{j} \frac{\Gamma(\alpha) t_{n}^{\alpha}}{\Gamma(1+\alpha)}\right. \\
& \left.+2 M+\lambda_{2} M \Delta t^{\alpha}+2 \lambda_{3} M \Delta t^{\alpha}\right) E_{\alpha}\left(2 \Gamma(\alpha) \lambda t_{n}^{\alpha}\right), \quad 1 \leq n \leq N,
\end{aligned}
$$

where $\quad \lambda=\lambda_{1}+\left(1 /\left(g_{0}^{(\alpha)}-g_{1}^{(\alpha)}\right) g_{0}^{(\alpha)}-g_{1}^{(\alpha)}\right) \lambda_{2}+\left(1 /\left(g_{1}^{(\alpha)}-\right.\right.$ $\left.\left.g_{2}^{(\alpha)}\right)\right) \lambda_{3}+\left(1 /\left(g_{m-1}^{(\alpha)}-g_{m}^{(\alpha)}\right)\right) \lambda_{4}+\left(1 /\left(g_{m}^{(\alpha)}-g_{m+1}^{(\alpha)}\right)\right) \lambda_{5}, \quad E_{\alpha}(z)$ $=\sum_{k=0}^{\infty}\left(z^{k} /(\Gamma(a+k \alpha))\right)$ is the Mittag-Leffler function, and $M=\max \left\{u^{-m}, u^{-m+1}, \cdots, u^{0}\right\}$.

Proof. By using the definition of ${ }^{R} D_{\Delta t}^{\alpha} u^{n}$ in (13), we have

$$
\begin{aligned}
\sum_{k=1}^{j} g_{j-k}^{(\alpha)} \delta_{t} u^{k}+g_{j}^{(\alpha)} u^{0} \leq & \Delta t^{\alpha}\left(\lambda_{1} u^{j}+\lambda_{2} u^{j-1}+\lambda_{3} u^{j-2}+\lambda_{4} u^{j-m}+\lambda_{5} u^{j-m-1}\right) \\
& +\Delta t^{\alpha} f^{j}
\end{aligned}
$$

Multiplying equation (18) by $\phi_{n-j}$ and summing the index $j$ from 1 to $n$, we get 


$$
\begin{aligned}
\sum_{j=1}^{n} \phi_{n-j} \sum_{k=1}^{j} g_{j-k}^{(\alpha)} \delta_{t} u^{k} \leq & \Delta t^{\alpha} \sum_{j=1}^{n} \phi_{n-j}\left(\lambda_{1} u^{j}+\lambda_{2} u^{j-1}+\lambda_{3} u^{j-2}+\lambda_{4} u^{j-m}+\lambda_{5} u^{j-m-1}\right) \\
& +\Delta t^{\alpha} \sum_{j=1}^{n} \phi_{n-j} f^{j}-\sum_{j=1}^{n} \phi_{n-j} g_{j}^{(\alpha)} u^{0}
\end{aligned}
$$

We change the order of summation and make use of the definition of $\phi_{n-j}$ to obtain

$$
\sum_{j=1}^{n} \phi_{n-j} \sum_{k=1}^{j} g_{j-k}^{(\alpha)} \delta_{t} u^{k}=\sum_{k=1}^{n} \delta_{t} u^{k} \sum_{j=1}^{k} \phi_{n-j} g_{j-k}^{(\alpha)}=\sum_{k=1}^{n} \delta_{t} u^{k}=u^{n}-u^{0}
$$

and using Lemma 4, we have

$$
\begin{aligned}
\Delta t^{\alpha} \sum_{j=1}^{n} \phi_{n-j} f^{j} & \leq \Delta t^{\alpha} \max _{1 \leq j \leq n} f^{j} \sum_{j=1}^{n} \phi_{n-j} \leq \Delta t^{\alpha} \max _{1 \leq j \leq n} f^{j} \frac{\Gamma(\alpha) n^{\alpha}}{\Gamma(1+\alpha)} \\
& =\max _{1 \leq j \leq n} f^{j} \frac{\Gamma(\alpha) t_{n}^{\alpha}}{\Gamma(1+\alpha)} .
\end{aligned}
$$

Noticing $g_{j}^{(\alpha)}$ is monotone decreasing and using Lemma 4 , we have

$$
-\sum_{j=1}^{n} \phi_{n-j} g_{j}^{(\alpha)} u^{0} \leq \sum_{j=1}^{n} \phi_{n-j} g_{j}^{(\alpha)} u^{0} \leq u^{0} \sum_{j=1}^{n} \phi_{n-j} g_{j-1}^{(\alpha)}=u^{0}
$$

Substituting (20), (21), and (22) into (19), we can obtain

$$
\begin{aligned}
u^{n} \leq & \Delta t^{\alpha} \sum_{j=1}^{n} \phi_{n-j}\left(\lambda_{1} u^{j}+\lambda_{2} u^{j-1}+\lambda_{3} u^{j-2}+\lambda_{4} u^{j-m}+\lambda_{5} u^{j-m-1}\right) \\
& +2 u^{0}+\max _{1 \leq j \leq n} f^{j} \frac{\Gamma(\alpha) t_{n}^{\alpha}}{\Gamma(1+\alpha)} .
\end{aligned}
$$

Applying Lemma 4, we have

$$
\begin{aligned}
\Delta t^{\alpha} \sum_{j=1}^{m} \phi_{n-j} u^{j-m} & \leq \frac{\Gamma(\alpha) t_{n}^{\alpha}}{\Gamma(1+\alpha)} M, \\
\Delta t^{\alpha} \sum_{j=1}^{m+1} \phi_{n-j} u^{j-m-1} & \leq \frac{\Gamma(\alpha) t_{n}^{\alpha}}{\Gamma(1+\alpha)} M .
\end{aligned}
$$

Therefore,

$$
\begin{aligned}
\lambda_{4} \Delta t^{\alpha} \sum_{j=1}^{m} \phi_{n-j} u^{j-m} & +\lambda_{5} \Delta t^{\alpha} \sum_{j=1}^{m+1} \phi_{n-j} u^{j-m-1}+2 u^{0}+\lambda_{2} \Delta t^{\alpha} \phi_{n-1} u^{0} \\
& +\lambda_{3} \Delta t^{\alpha}\left(\phi_{n-1} u^{-1}+\phi_{n-2} u^{0}\right) \leq \lambda_{4} \frac{\Gamma(\alpha) t_{n}^{\alpha}}{\Gamma(1+\alpha)} M \\
& +\lambda_{5} \frac{\Gamma(\alpha) t_{n}^{\alpha}}{\Gamma(1+\alpha)} M+2 M+\lambda_{2} M \Delta t^{\alpha}+2 \lambda_{3} M \Delta t^{\alpha} .
\end{aligned}
$$

\section{Denote}

$$
\begin{aligned}
\Psi_{n}= & \lambda_{4} \frac{\Gamma(\alpha) t_{n}^{\alpha}}{\Gamma(1+\alpha)} M+\lambda_{5} \frac{\Gamma(\alpha) t_{n}^{\alpha}}{\Gamma(1+\alpha)} M+\max _{1 \leq j \leq n} f^{j} \frac{\Gamma(\alpha) t_{n}^{\alpha}}{\Gamma(1+\alpha)} \\
& +2 M+\lambda_{2} M \Delta t^{\alpha}+2 \lambda_{3} M \Delta t^{\alpha}
\end{aligned}
$$

Equation (23) can be rewritten as

$$
\begin{aligned}
\left(1-\lambda_{1} \Delta t^{\alpha}\right) u^{n} \leq & \lambda_{1} \Delta t^{\alpha} \sum_{j=1}^{n-1} \phi_{n-j} u^{j}+\lambda_{2} \Delta t^{\alpha} \sum_{j=2}^{n} \phi_{n-j} u^{j-1} \\
& +\lambda_{3} \Delta t^{\alpha} \sum_{j=3}^{n} \phi_{n-j} u^{j-2}+\lambda_{4} \Delta t^{\alpha} \sum_{j=m+1}^{n} \phi_{n-j} u^{j-m} \\
& +\lambda_{5} \Delta t^{\alpha} \sum_{j=m+2}^{n} \phi_{n-j} u^{j-m-1}+\Psi_{n} .
\end{aligned}
$$

Let $\Delta t^{*}=\sqrt[\alpha]{1 /\left(2 \lambda_{1}\right)}$, when $\Delta t \leq \Delta t^{*}$, we have

$$
\begin{aligned}
u^{n} \leq & 2 \Psi_{n}+2 \Delta t^{\alpha}\left[\lambda_{1} \sum_{j=1}^{n-1} \phi_{n-j} u^{j}+\lambda_{2} \sum_{j=2}^{n} \phi_{n-j} u^{j-1}+\lambda_{3} \sum_{j=3}^{n} \phi_{n-j} u^{j-2}\right. \\
& \left.+\lambda_{4} \sum_{j=m+1}^{n} \phi_{n-j} u^{j-m}+\lambda_{5} \sum_{j=m+2}^{n} \phi_{n-j} u^{j-m-1}\right] .
\end{aligned}
$$

Let $V=\left(u^{n}, u^{n-1}, \cdots, u^{1}\right)^{T}$, then (28) can be rewritten in the following matrix form:

$$
V \leq 2 \Psi_{n} \overleftarrow{e}+\left(\lambda_{1} W_{1}+\lambda_{2} W_{2}+\lambda_{3} W_{3}+\lambda_{4} W_{4}+\lambda_{5} W_{5}\right) V
$$

where

$$
W_{1}=2(\Delta t)^{\alpha}\left(\begin{array}{cccccc}
0 & \phi_{1} & \phi_{2} & \cdots & \phi_{n-2} & \phi_{n-1} \\
0 & 0 & \phi_{1} & \cdots & \phi_{n-3} & \phi_{n-2} \\
\vdots & \vdots & \vdots & \ddots & \vdots & \vdots \\
0 & 0 & 0 & \cdots & \phi_{1} & \phi_{2} \\
0 & 0 & 0 & \cdots & 0 & \phi_{1} \\
0 & 0 & 0 & 0 & \cdots & 0
\end{array}\right)_{n \times n}
$$


$W_{2}=2(\Delta t)^{\alpha}\left(\begin{array}{cccccc}0 & \phi_{0} & \phi_{1} & \cdots & \phi_{n-3} & \phi_{n-2} \\ 0 & 0 & \phi_{0} & \cdots & \phi_{n-4} & \phi_{n-3} \\ \vdots & \vdots & \vdots & \ddots & \vdots & \vdots \\ 0 & 0 & 0 & \cdots & \phi_{0} & \phi_{1} \\ 0 & 0 & 0 & \cdots & 0 & \phi_{0} \\ 0 & 0 & 0 & 0 & \cdots & 0 \\ & & & & & \end{array}\right)_{n \times n}$

$W_{3}=2(\Delta t)^{\alpha}\left(\begin{array}{cccccc}0 & 0 & \phi_{0} & \cdots & \phi_{n-4} & \phi_{n-3} \\ 0 & 0 & 0 & \cdots & \phi_{n-5} & \phi_{n-4} \\ \vdots & \vdots & \vdots & \ddots & \vdots & \vdots \\ 0 & 0 & 0 & \cdots & 0 & \phi_{0} \\ 0 & 0 & 0 & \cdots & 0 & 0 \\ 0 & 0 & 0 & 0 & 0 & 0\end{array}\right)_{n \times n}$

$W_{4}=2(\Delta t)^{\alpha}\left(\begin{array}{cccccccc}0 & \cdots & 0 & \phi_{0} & \phi_{1} & \cdots & \phi_{n-m-2} & \phi_{n-m-1} \\ 0 & \cdots & 0 & 0 & \phi_{0} & \cdots & \phi_{n-m-3} & \phi_{n-m-2} \\ \vdots & \cdots & \vdots & \vdots & \vdots & \ddots & \vdots & \vdots \\ 0 & \cdots & 0 & 0 & 0 & \cdots & \phi_{0} & \phi_{1} \\ 0 & \cdots & 0 & 0 & 0 & \cdots & 0 & \phi_{0} \\ 0 & & 0 & 0 & 0 & \cdots & 0 & 0 \\ \vdots & \cdots & \vdots & \vdots & \vdots & \cdots & \vdots & \vdots \\ 0 & \cdots & 0 & 0 & 0 & \cdots & 0 & 0 \\ & & & & & & & \end{array}\right)_{n \times n}$

$W_{5}=2(\Delta t)^{\alpha}\left(\begin{array}{cccccccc}0 & \cdots & 0 & 0 & \phi_{0} & \cdots & \phi_{n-m-3} & \phi_{n-m-2} \\ 0 & \cdots & 0 & 0 & 0 & \cdots & \phi_{n-m-4} & \phi_{n-m-3} \\ \vdots & \cdots & \vdots & \vdots & \vdots & \ddots & \vdots & \vdots \\ 0 & \cdots & 0 & 0 & 0 & \cdots & 0 & \phi_{0} \\ 0 & \cdots & 0 & 0 & 0 & \cdots & 0 & 0 \\ 0 & & 0 & 0 & 0 & \cdots & 0 & 0 \\ \vdots & \cdots & \vdots & \vdots & \vdots & \cdots & \vdots & \vdots \\ 0 & \cdots & 0 & 0 & 0 & \cdots & 0 & 0 \\ & & & & & & & \end{array}\right)_{n \times n}$

Since the definition of $\phi_{n}$, we have

$$
\phi_{n-j} \leq \frac{1}{g_{j-1}^{(\alpha)}-g_{j}^{(\alpha)}} \phi_{n}
$$

Then,

$$
\begin{aligned}
& W_{2} V \leq \frac{1}{g_{0}^{(\alpha)}-g_{1}^{(\alpha)}} W_{1} V, \\
& W_{3} V \leq \frac{1}{g_{1}^{(\alpha)}-g_{2}^{(\alpha)}} W_{1} V, \\
& W_{4} V \leq \frac{1}{g_{m-1}^{(\alpha)}-g_{m}^{(\alpha)}} W_{1} V, \\
& W_{5} V \leq \frac{1}{g_{m}^{(\alpha)}-g_{m+1}^{(\alpha)}} W_{1} V .
\end{aligned}
$$

Hence, (29) can be shown as follows

$$
\begin{aligned}
V \leq & \left(\lambda_{1}+\frac{1}{g_{0}^{(\alpha)}-g_{1}^{(\alpha)}} \lambda_{2}+\frac{1}{g_{1}^{(\alpha)}-g_{2}^{(\alpha)}} \lambda_{3}+\frac{1}{g_{m-1}^{(\alpha)}-g_{m}^{(\alpha)}} \lambda_{4}\right. \\
& \left.+\frac{1}{g_{m}^{(\alpha)}-g_{m+1}^{(\alpha)}} \lambda_{5}\right) W_{1} V+2 \Psi_{n}^{\overleftarrow{e}}=W V+2 \Psi_{n} \overleftarrow{e}
\end{aligned}
$$

where $W=\lambda W_{1}$.

Therefore,

$$
\begin{aligned}
V & \leq W V+2 \Psi_{n} \overleftarrow{e} \leq W\left(W V+2 \Psi_{n} \overleftarrow{e}\right)+2 \Psi_{n} \overleftarrow{e} \\
& =W^{2} V+2 \Psi_{n} \sum_{j=0}^{1} W^{j} \overleftarrow{e} \leq \cdots \leq W^{n} V+2 \Psi_{n} \sum_{j=0}^{n-1} W^{j} \overleftarrow{e}
\end{aligned}
$$

According to Lemma 5, the result can be proved.

Lemma 7 (see [51]). For any sequence $\left\{e^{k}\right\}_{k=0}^{N} \subset X_{h}$, the following inequality holds:

$$
\left\langle{ }^{R} D_{\Delta t}^{\alpha} e^{k},\left(1-\frac{\alpha}{2}\right) e^{k}+\frac{\alpha}{2} e^{k-1}\right\rangle \geq \frac{1^{R}}{2} D_{\Delta t}^{\alpha}\left\|e^{k}\right\|^{2}, \quad 1 \leq k \leq N .
$$

Lemma 8 (see [52]). There exists a positive constant $C_{\Omega}$, independent of $h$, for any $v \in H^{s}(\Omega) \cap H_{0}^{1}(\Omega)$, such that

$$
\left\|v-R_{h} v\right\|_{L^{2}}+h\left\|\nabla\left(v-R_{h} v\right)\right\|_{L^{2}} \leq C_{\Omega} h^{s}\|v\|_{H^{s}}, \quad 1 \leq s \leq r+1 .
$$

3.2. Proof of Theorem 1. Now, we are ready to prove our main results.

Proof. Taking $t=t_{n-(\alpha / 2)}$ in the first equation (1), we can find that $u^{n}$ satisfies the following equation:

$$
\left\langle{ }^{R} D_{\Delta t}^{\alpha} u^{n}, v\right\rangle+\left\langle\nabla u^{n, \alpha}, \nabla v\right\rangle=\left\langle f\left(t_{n-(\alpha / 2)}, u \wedge^{n, \alpha}, u^{n-m_{\tau}, \alpha}\right), v\right\rangle+\left\langle P^{n}, v\right\rangle,
$$


for $n=1,2,3, \cdots, N$ and $\forall v \in X_{h}$, where

$$
\begin{aligned}
P^{n}= & { }^{R} D_{\Delta t}^{\alpha} u^{n}-{ }^{R} D_{t_{n-(\alpha / 2)}^{\alpha}}^{\alpha} u+\Delta u^{n-(\alpha / 2)}-\Delta u^{n, \alpha} \\
& +f\left(t_{n-(\alpha / 2)}, u^{n-(\alpha / 2)}, u^{n-m_{\tau}-(\alpha / 2)}\right) \\
& -f\left(t_{n-(\alpha / 2)}, u \wedge^{n, \alpha}, u^{n-m_{\tau}, \alpha}\right) .
\end{aligned}
$$

Now, we estimate the error of $\left\|P^{n}\right\|$. Actually, from the definition of $u^{n, \alpha}$ and $u \wedge^{n, \alpha}$ and the regularity of the exact solution (8), we can obtain that

$$
\begin{aligned}
\left\|u^{n-(\alpha / 2)}-u^{n, \alpha}\right\| & =\left\|\left(1-\frac{\alpha}{2}\right) u^{n-(\alpha / 2)}+\frac{\alpha}{2} u^{n-(\alpha / 2)}-\left(1-\frac{\alpha}{2}\right) u^{n-(\alpha / 2)} u^{n-1}\right\| \\
& =\left\|\left(1-\frac{\alpha}{2}\right)\left(u^{n-(\alpha / 2)-u^{n}}\right)+\frac{\alpha}{2}\left(u^{n-(\alpha / 2)-u^{n-1}}\right)\right\| \\
& =\left\|-\left(1-\frac{\alpha}{2}\right)+\frac{\alpha}{2} \Delta t u^{\prime}\left(\xi_{1}\right)+\left(1-\frac{\alpha}{2}\right)+\frac{\alpha}{2} \Delta t u^{\prime}\left(\xi_{2}\right)\right\| \\
& =\left(1-\frac{\alpha}{2}\right)+\frac{\alpha}{2} \Delta t\left\|u^{\prime}\left(\xi_{2}\right)-u^{\prime}\left(\xi_{1}\right)\right\| \\
& \leq\left(1-\frac{\alpha}{2}\right)+\frac{\alpha}{2} \Delta t \int_{t_{n-1}}^{t_{n}}\left\|u_{t t}(s)\right\| d s \leq C_{1} \Delta t^{2},
\end{aligned}
$$

$$
\begin{aligned}
\left\|u^{n-(\alpha / 2)}-u \wedge^{n, \alpha}\right\|= & \left\|u^{n-(\alpha / 2)}-\left(2-\frac{\alpha}{2}\right) u^{n-1}+\left(1-\frac{\alpha}{2}\right) u^{n-2}\right\| \\
= & \|\left(2-\frac{\alpha}{2}\right) u^{n-(\alpha / 2)}-\left(2-\frac{\alpha}{2}\right) u^{n-1} \\
& +\left(1-\frac{\alpha}{2}\right) u^{n-2}-\left(1-\frac{\alpha}{2}\right) u^{n-(\alpha / 2)} \| \\
= & \|\left(2-\frac{\alpha}{2}\right)\left(u^{n-(\alpha / 2)}-u^{n-1}\right) \\
& +\left(1-\frac{\alpha}{2}\right)\left(u^{n-2}-u^{n-(\alpha / 2)}\right) \| \\
= & \|\left(2-\frac{\alpha}{2}\right)\left(1-\frac{\alpha}{2}\right) \Delta t u^{\prime}\left(\xi_{3}\right) \\
& -\left(2-\frac{\alpha}{2}\right)\left(1-\frac{\alpha}{2}\right) \Delta t u^{\prime}\left(\xi_{4}\right) \| \\
= & \left(2-\frac{\alpha}{2}\right)\left(1-\frac{\alpha}{2}\right) \Delta t\left\|u^{\prime}\left(\xi_{3}\right)-u^{\prime}\left(\xi_{4}\right)\right\| \\
\leq & \left(2-\frac{\alpha}{2}\right)\left(1-\frac{\alpha}{2}\right) \Delta t \int_{t_{n-2}}^{t_{n-1}}\left\|u_{t t}(s)\right\| d s \leq C_{2} \Delta t^{2},
\end{aligned}
$$

where $\xi_{1} \in\left(t_{n-(\alpha / 2)}, t_{n}\right), \xi_{2} \in\left(t_{n-1}, t_{n-(\alpha / 2)}\right), \xi_{3} \in\left(t_{n-(\alpha / 2)}, t_{n-1}\right)$ , $\xi_{4} \in\left(t_{n-2}, t_{n-(\alpha / 2)}\right), C_{1}=(1-(\alpha / 2))(\alpha / 2) K, C_{2}=(2-(\alpha / 2))$ $(1-(\alpha / 2)) K$ are constants.

Applying (39) and (40) and the Lipschitz condition,

$$
\begin{aligned}
& \left\|f\left(t_{n-(\alpha / 2)}, u^{n-(\alpha / 2)}, u^{n-m_{\tau}-(\alpha / 2)}\right)-f\left(t_{n-(\alpha / 2)}, u \wedge^{n, \alpha}, u^{n-m_{\tau}, \alpha}\right)\right\| \\
& \quad \leq\left(L_{1} C_{1}+L_{2} C_{2}\right) \Delta t^{2},
\end{aligned}
$$

$$
\left\|\Delta\left(u^{n, \alpha}-u^{n-(\alpha / 2)}\right)\right\| \leq C_{1} \Delta t^{2}
$$

which further implies that

$$
\left\|P^{n}\right\| \leq C_{K}(\Delta t)^{2}, \quad n=1,2,3, \cdots, N
$$

here $C_{K}=L_{1} C_{1}+L_{2} C_{2}$.

Denote $\theta_{h}^{n}=R_{h} u^{n}-U_{h}^{n}, n=0,1, \cdots, N$.

Substituting fully scheme (5) from equation (37) and using the property in (7), we can get that

$\left\langle{ }^{R} D_{\Delta t}^{\alpha} \theta_{h}^{n}, v\right\rangle+\left\langle\nabla \theta_{h}^{n, \alpha}, v\right\rangle=\left\langle R_{1}^{n}, v\right\rangle+\left\langle P^{n}, v\right\rangle-\left\langle{ }^{R} D_{\Delta t}^{\alpha}\left(u^{n}-R_{h} u^{n}\right), v\right\rangle$,

where

$$
R_{1}^{n}=f\left(t_{n-(\alpha / 2)}, \widehat{U}_{h}^{n, \alpha}, U_{h}^{n-m_{\tau}, \alpha}\right)-f\left(t_{n-(\alpha / 2)}, u \wedge^{n, \alpha}, u^{n-m_{\tau}, \alpha}\right) .
$$

Setting $v=\theta_{h}^{n, \alpha}$ and applying the Cauchy-Schwarz inequality, it holds that

$$
\begin{aligned}
\left\langle{ }^{R} D_{\Delta t}^{\alpha} \theta_{h}^{n}, \theta_{h}^{n, \alpha}\right\rangle+ & \left\|\nabla \theta_{h}^{n, \alpha}\right\|^{2} \leq\left\|R_{1}^{n}\right\|\left\|\theta_{h}^{n, \alpha}\right\|+\left\|P^{n}\right\|\left\|\theta_{h}^{n, \alpha}\right\| \\
& +\left\|^{R} D_{\Delta t}^{\alpha}\left(u^{n}-R_{h} u^{n}\right)\right\|\left\|\theta_{h}^{n, \alpha}\right\| .
\end{aligned}
$$

Noticing the fact $a b \leq 1 / 2\left(a^{2}+b^{2}\right)$ and $\left\|\nabla \theta_{h}^{n, \alpha}\right\|^{2} \geq 0$,

$$
\begin{aligned}
\left\langle{ }^{R} D_{\Delta t}^{\alpha} \theta_{h}^{n}, \theta_{h}^{n, \alpha}\right\rangle \leq & \frac{1}{2}\left(\left\|R_{1}^{n}\right\|^{2}+\left\|P^{n}\right\|^{2}+\left\|^{R} D_{\Delta t}^{\alpha}\left(u^{n}-R_{h} u^{n}\right)\right\|^{2}\right) \\
& +\frac{3}{2}\left\|\theta_{h}^{n, \alpha}\right\|^{2} .
\end{aligned}
$$

Together with (9) and (36), we can arrive that

$$
\left\|^{R} D_{\Delta t}^{\alpha}\left(u^{n}-R_{h} u^{n}\right)\right\| \leq C_{\Omega} h^{r+1}\left\|^{R} D_{\Delta t}^{\alpha} u^{n}\right\|_{H^{r+1}} \leq C_{\Omega} K h^{r+1} .
$$

$$
\begin{aligned}
\left\|u \wedge^{n, \alpha}-R_{h} u \wedge^{n, \alpha}\right\|= & \|\left(2-\frac{\alpha}{2}\right) u^{n-1}-\left(1-\frac{\alpha}{2}\right) u^{n-2} \\
& -\left(2-\frac{\alpha}{2}\right) R_{h} u^{n-1}+\left(1-\frac{\alpha}{2}\right) R_{h} u^{n-2} \| \\
\leq & \left(2-\frac{\alpha}{2}\right)\left\|u^{n-1}-R_{h} u^{n-1}\right\|+\left(1-\frac{\alpha}{2}\right)\left\|u^{n-2}-R_{h} u^{n-2}\right\| \\
\leq & \left(2-\frac{\alpha}{2}\right) C_{\Omega} h^{r+1}\left\|u^{n-1}\right\|_{H^{r+1}} \\
& +\left(1-\frac{\alpha}{2}\right) C_{\Omega} h^{r+1}\left\|u^{n-2}\right\|_{H^{r+1}} \\
\leq & \left(2-\frac{\alpha}{2}\right) C_{\Omega} K h^{r+1}+\left(1-\frac{\alpha}{2}\right) C_{\Omega} K h^{r+1} \leq C_{3} h^{r+1} .
\end{aligned}
$$


Similarly, we have

$$
\begin{aligned}
\left\|u^{n-m_{\tau}, \alpha}-R_{h} u^{n-m_{\tau}, \alpha}\right\|= & \|\left(1-\frac{\alpha}{2}\right) u^{n-m_{\tau}}+\frac{\alpha}{2} u^{n-m_{\tau}-1} \\
& -\left(1-\frac{\alpha}{2}\right) R_{h} u^{n-m_{\tau}}-\frac{\alpha}{2} R_{h} u^{n-m_{\tau}-1} \| \\
\leq & \left(1-\frac{\alpha}{2}\right) C_{\Omega} K h^{r+1}+\frac{\alpha}{2} C_{\Omega} K h^{r+1} \\
\leq & C_{4} h^{r+1},
\end{aligned}
$$

where $C_{3}=2(2-(\alpha / 2)) C_{\Omega} K, C_{4}=2 \max \{(1-(\alpha / 2)),(\alpha / 2)$ \}$C_{\Omega} K$.

Therefore,

$$
\begin{aligned}
\left\|R_{1}^{n}\right\|= & \left\|f\left(t_{n-(\alpha / 2)}, u \wedge^{n, \alpha}, u^{n-m_{\tau}, \alpha}\right)-f\left(t_{n-(\alpha / 2)}, \widehat{U}_{h}^{n, \alpha}, U_{h}^{n-m_{\tau}, \alpha}\right)\right\| \\
\leq & L_{1}\left\|u \wedge^{n, \alpha}-\widehat{U}_{h}^{n, \alpha}\right\|+L_{2}\left\|u^{n-m_{\tau}, \alpha}-U_{h}^{n-m_{\tau}, \alpha}\right\| \\
\leq & L_{1}\left\|\widehat{\theta}_{h}^{n, \alpha}\right\|+L_{2}\left\|\theta_{h}^{n-m_{\tau}, \alpha}\right\|+L_{1}\left\|u \wedge^{n, \alpha}-R_{h} u \wedge^{n, \alpha}\right\| \\
& +L_{2}\left\|u^{n-m_{\tau}, \alpha}-R_{h} u^{n-m_{\tau}, \alpha}\right\| \leq L_{1}\left\|\widehat{\theta}_{h}^{n, \alpha}\right\|+L_{2}\left\|\theta_{h}^{n-m_{\tau}, \alpha}\right\| \\
& +\left(L_{1} C_{3}+L_{2} C_{4}\right) h^{r+1} .
\end{aligned}
$$

Substituting (42), (47), and (50) into (46) and the fact $(a+b+c)^{2} \leq 3 a^{2}+3 b^{2}+3 c^{2}$, we can get

$$
\begin{aligned}
\left\langle{ }^{R} D_{\Delta t}^{\alpha} \theta_{h}^{n}, \theta_{h}^{n, \alpha}\right\rangle \leq & \frac{3}{2}\left\|\theta_{h}^{n, \alpha}\right\|^{2}+\frac{3 L_{1}^{2}}{2}\left\|\widehat{\theta}_{h}^{n, \alpha}\right\|^{2}+\frac{3 L_{2}^{2}}{2}\left\|\theta_{h}^{n-m_{\tau}, \alpha}\right\|^{2} \\
& +\frac{C_{K}^{2}}{2}(\Delta t)^{4}+\frac{1}{2}\left[3\left(L_{1}^{2} C_{3}^{2}+L_{2}^{2} C_{4}^{2}\right)+\left(C_{K} K\right)^{2}\right] h^{2(r+1)} \\
\leq & \frac{3}{2}\left\|\theta_{h}^{n, \alpha}\right\|^{2}+\frac{3 L_{1}^{2}}{2}\left\|\widehat{\theta}_{h}^{n, \alpha}\right\|^{2}+\frac{3 L_{2}^{2}}{2}\left\|\theta_{h}^{n-m_{\tau}, \alpha}\right\|^{2} \\
& +\frac{C_{4}}{2}\left(\Delta t^{2}+h^{r+1}\right)^{2},
\end{aligned}
$$

where $C_{4}=\max \left\{C_{K}^{2}, 3\left(L_{1}^{2} C_{3}^{2}+L_{2}^{2} C_{4}^{2}\right)+\left(C_{K} K\right)^{2}\right\}$.

Applying Lemma 7, we have

${ }^{R} D_{\Delta t}^{\alpha}\left\|\theta_{h}^{n}\right\|^{2} \leq 3\left\|\theta_{h}^{n, \alpha}\right\|^{2}+3 L_{1}^{2}\left\|\widehat{\theta}_{h}^{n, \alpha}\right\|^{2}+3 L_{2}^{2}\left\|\theta_{h}^{n-m_{\tau}, \alpha}\right\|^{2}+C_{4}\left(\Delta t^{2}+h^{r+1}\right)^{2}$.
TABLE 1: The errors and convergence orders in temporal direction by using Q-FEM.

\begin{tabular}{lcccc}
\hline \multirow{2}{*}{$M$} & \multicolumn{2}{c}{$\alpha=0.4$} & \multicolumn{2}{c}{$\alpha=0.6$} \\
& Errors & Orders & Errors & Orders \\
\hline 5 & $1.6856 e-03$ & $*$ & $5.3999 e-03$ & $*$ \\
10 & $2.9420 e-04$ & 2.5184 & $1.2503 e-03$ & 2.1106 \\
20 & $5.9619 e-05$ & 2.3030 & $3.0266 e-04$ & 2.0465 \\
40 & $1.3851 e-05$ & 2.1058 & $7.4700 e-05$ & 2.0185 \\
\hline
\end{tabular}

In terms of the definition of $\left\|\theta_{h}^{n, \alpha}\right\|$ and $\widehat{\theta}_{h}^{n, \alpha}$, we obtain

$$
\begin{aligned}
{ }^{R} D_{\Delta t}^{\alpha}\left\|\theta_{h}^{n}\right\|^{2} \leq & 3\left(1-\frac{\alpha}{2}\right)^{2}\left\|\theta_{h}^{n}\right\|^{2}+\left(3\left(\frac{\alpha}{2}\right)^{2}+3 L_{1}^{2}\left(2-\frac{\alpha}{2}\right)^{2}\right)\left\|\theta_{h}^{n-1}\right\|^{2} \\
& +3 L_{1}^{2}\left(1-\frac{\alpha}{2}\right)^{2}\left\|\theta_{h}^{n-2}\right\|^{2}+3 L_{2}^{2}\left(1-\frac{\alpha}{2}\right)^{2}\left\|\theta_{h}^{n-m_{\tau}}\right\|^{2} \\
& +3 L_{2}^{2}\left(\frac{\alpha}{2}\right)^{2}\left\|\theta_{h}^{n-m_{\tau}-1}\right\|^{2}+C_{4}\left(\Delta t^{2}+h^{r+1}\right)^{2} .
\end{aligned}
$$

Using Theorem 6, we can find a positive constant $\Delta t^{*}$ such that $\Delta t \leq \Delta t^{*}$, then

$$
\left\|\theta_{h}^{n}\right\|^{2} \leq C_{5}\left(\Delta t^{2}+h^{r+1}\right)^{2}
$$

where $C_{5}$ is a nonnegative constant which only depends on $L_{1}, L_{2}, C_{4}, C_{K}, C_{\Omega}$. In terms of the definition of $\theta_{h}^{n}$, we have

$$
\left\|u^{n}-U_{h}^{n}\right\| \leq\left\|u^{n}-R_{h} u^{n}\right\|+\left\|R_{h} u^{n}-U_{h}^{n}\right\| \leq C_{1}^{*}\left(\Delta t^{2}+h^{r+1}\right) .
$$

Then, we complete the proof.

\section{Numerical Examples}

In this section, we give two examples to verify our theoretical results. The errors are all calculated in L2-norm.

Example 1. Consider the nonlinear time-fractional MackeyGlass-type equation

$$
\left\{\begin{array}{l}
{ }^{R} D_{t}^{\alpha} u(x, y, t)=\Delta u(x, y, t)-2 u(x, y, t)+\frac{u(x, y, t-0.1)}{1+u^{2}(x, y, t-0.1)}+f(x, y, t), \quad(x, y) \in[0,1]^{2}, t \in[0,1], \\
u(x, y, t)=t^{2} \sin (\pi x) \sin (\pi y), \quad(x, y) \in[0,1]^{2}, t \in[-0.1,0],
\end{array}\right.
$$


TABLE 2: The errors and convergence orders in spatial direction by using L-FEM.

\begin{tabular}{lcccc}
\hline \multirow{2}{*}{$M$} & \multicolumn{2}{c}{$\alpha=0.4$} & \multicolumn{2}{c}{$\alpha=0.6$} \\
& Errors & Orders & Errors & Orders \\
\hline 5 & $7.2603 e-02$ & $*$ & $7.2065 e-02$ & $*$ \\
10 & $1.9449 e-02$ & 1.9003 & $1.9297 e-02$ & 1.9009 \\
20 & $8.7594 e-03$ & 1.9673 & $8.6948 e-03$ & 1.9662 \\
40 & $4.9508 e-03$ & 1.9834 & $4.9180 e-03$ & 1.9807 \\
\hline
\end{tabular}

TABLE 3: The errors and convergence orders in spatial direction by using Q-FEM.

\begin{tabular}{lcccc}
\hline \multirow{2}{*}{$M$} & \multicolumn{2}{c}{$\alpha=0.4$} & \multicolumn{2}{c}{$\alpha=0.6$} \\
& Errors & Orders & Errors & Orders \\
\hline 5 & $2.0750 e-03$ & $*$ & $2.0746 e-03$ & $*$ \\
10 & $2.4888 e-04$ & 3.0596 & $2.5148 e-04$ & 3.0443 \\
20 & $7.3251 e-05$ & 3.0165 & $7.5802 e-05$ & 2.9577 \\
40 & $3.0946 e-05$ & 2.9952 & $3.4200 e-05$ & 2.7666 \\
\hline
\end{tabular}

where

$$
\begin{aligned}
f(x, y, t)= & \frac{2 t^{2-\alpha}}{\Gamma(3-\alpha)} \sin (\pi x) \sin (\pi y)+2 t^{2} \pi^{2} \sin (\pi x) \sin (\pi y) \\
& -2 t^{2} \sin (\pi x) \sin (\pi y) \\
& -\frac{(t-0.1)^{2} \sin (\pi x) \sin (\pi y)}{1+\left[(t-0.1)^{2} \sin (\pi x) \sin (\pi y)\right]^{2}} .
\end{aligned}
$$

The exact solution is given as

$$
u(x, t)=t^{2} \sin (\pi x) \sin (\pi y)
$$

TABLE 4: The errors and orders in temporal and spatial direction by using L-FEM.

\begin{tabular}{lcccc}
\hline \multirow{2}{*}{$M$} & \multicolumn{2}{c}{$\alpha=0.4$} & \multicolumn{2}{c}{$\alpha=0.6$} \\
& Errors & Orders & Errors & Orders \\
\hline 5 & $8.3275 e-02$ & $*$ & $8.3375 e-02$ & $*$ \\
10 & $2.2615 e-02$ & 1.8806 & $2.2732 e-02$ & 1.8749 \\
20 & $5.8356 e-03$ & 1.9543 & $5.8662 e-03$ & 1.9542 \\
40 & $1.4707 e-03$ & 1.9884 & $1.4784 e-03$ & 1.9884 \\
\hline
\end{tabular}

TABle 5: The errors and orders in temporal direction and spatial direction by using Q-FEM.

\begin{tabular}{cccccc}
\hline & \multirow{2}{*}{$M$} & \multicolumn{2}{c}{$\alpha=0.4$} & \multicolumn{2}{c}{$\alpha=0.6$} \\
& 8 & Errors & Orders & Errors & Orders \\
\hline \multirow{4}{*}{$N=M^{(3 / 2)}$} & $6.7379 e-04$ & $*$ & $6.9141 e-04$ & $*$ \\
& 10 & $3.1416 e-04$ & 3.0459 & $3.4945 e-04$ & 3.0579 \\
& 12 & $1.9415 e-04$ & 3.0968 & $1.9787 e-04$ & 3.1196 \\
& 14 & $1.1891 e-04$ & 3.1806 & $1.1992 e-04$ & 3.2485 \\
\hline
\end{tabular}

In order to test the convergence order in temporal direction, we fixed $M=40$ for $\alpha=0.4, \alpha=0.6$ and different $N$. Similarly, to obtain the convergence order in spatial direction, we fixed $N=100$ for $\alpha=0.4, \alpha=0.6$, and different $M$. Table 1 gives the errors and convergence orders in temporal direction by using the Q-FEM. Table 1 shows that the convergence order in temporal direction is 2. Similarly, Tables 2 and 3 give the errors and convergence orders in spatial direction by using the L-FEM and Q-FEM, respectively. These numerical results correspond to our theoretical convergence order.

Example 2. Consider the following nonlinear time-fractional Nicholson's blowflies equation

$$
\left\{\begin{array}{l}
{ }^{R} D_{t}^{\alpha} u(x, y, z, t)=\Delta u(x, y, z, t)-2 u(x, y, z, t)+u(x, y, z, t-0.1) \exp \{-u(x, y, z, t-0.1)\}+f(x, y, z, t), \quad(x, y, z) \in[0,1]^{3}, t \in[0,1], \\
u(x, y, z, t)=t^{2} \sin (\pi x) \sin (\pi y) \sin (\pi z), \quad(x, y, z) \in[0,1]^{3}, t \in[-0.1,0],
\end{array}\right.
$$

where

$$
\begin{aligned}
f(x, y, z, t)= & \left(2 t^{2-\alpha} / \Gamma(3-\alpha)\right) \sin (\pi x) \sin (\pi y) \sin (\pi z) \\
& +2 t^{2}\left(\pi^{2}-1\right) \sin (\pi x) \sin (\pi y) \sin (\pi z) \\
& -(-0.1)^{2} \sin (\pi x) \sin (\pi y) \sin (\pi z) \exp \\
& \cdot\left\{-(t-0.1)^{2} \sin (\pi x) \sin (\pi y) \sin (\pi z)\right\},
\end{aligned}
$$

the exact solution is given as

$$
u(x, t)=t^{2} \sin (\pi x) \sin (\pi y) \sin (\pi z)
$$

In this example, in order to test the convergence order in temporal and spatial direction, we solve this problem by using the L-FEM with $M=N$ and the Q-FEM with $N=$ $M^{(3 / 2)}$, respectively. Tables 4 and 5 show that the convergence orders in temporal and spatial direction are 2 and 3 , 
respectively. The numerical results confirm our theoretical convergence order.

\section{Conclusions}

We proposed a linearized fractional Crank-NicolsonGalerkin FEM for the nonlinear fractional parabolic equations with time delay. A novel fractional Grönwall-type inequality is developed. With the help of the inequality, we prove convergence of the numerical scheme. Numerical examples confirm our theoretical results.

\section{Data Availability}

The data used to support the findings of this study are included within the article.

\section{Conflicts of Interest}

The authors declare that they have no conflicts of interest.

\section{Acknowledgments}

This work is supported by the Natural Science Foundation of Hunan Province (Grant No. 2018JJ3628) and the National Natural Science Foundation of China (Grant Nos.12071488 and 11971488).

\section{References}

[1] F. Höfling and T. Franosch, "Anomalous transport in the crowded world of biological cells," Reports on Progress in Physics, vol. 76, no. 4, article 046602, 2013.

[2] A. Arafa, S. Rida, and M. Khalil, "Fractional modeling dynamics of HIV and CD4+ T-cells during primary infection," Nonlinear Biomedical Physics, vol. 6, no. 1, p. 1, 2012.

[3] R. L. Magin, Fractional Calculus in Bioengineering, Begell House Redding, 2006.

[4] N. Sebaa, Z. E. A. Fellah, W. Lauriks, and C. Depollier, “Application of fractional calculus to ultrasonic wave propagation in human cancellous bone," Signal Processing, vol. 86, no. 10, pp. 2668-2677, 2006.

[5] A. Carpinteri and F. Mainardi, Fractals and Fractional Calculus in Continuum Mechanics, vol. 378, Springer, 2014.

[6] B. West, M. Bologna, and P. Grigolini, Physics of Fractal Operators, Springer Science, Business Media, 2012.

[7] D. Li and C. Zhang, "Long time numerical behaviors of fractional pantograph equations," Mathematics and Computers in Simulation, vol. 172, pp. 244-257, 2020.

[8] A. A. A. Kilbas, H. M. Srivastava, and J. J. Trujillo, Theory and Applications of Fractional Differential Equations, vol. 204, Elsevier Science Limited, 2006.

[9] Q. Zhang, Y. Ren, X. Lin, and Y. Xu, "Uniform convergence of compact and BDF methods for the space fractional semilinear delay reaction-diffusion equations," Applied Mathematics and Computation, vol. 358, pp. 91-110, 2019.

[10] W. Chen, L. Ye, and H. Sun, "Fractional diffusion equations by the Kansa method," Computers \& Mathematcs with Applications, vol. 59, no. 5, pp. 1614-1620, 2010.

[11] M. Dehghan, M. Abbaszadeh, and A. Mohebbi, "Error estimate for the numerical solution of fractional reaction- subdiffusion process based on a meshless method," Journal of Computational and Applied Mathematics, vol. 280, pp. 1436, 2015.

[12] D. A. Murio, "Implicit finite difference approximation for time fractional diffusion equations," Computers \& Mathematics with Applications, vol. 56, no. 4, pp. 1138-1145, 2008.

[13] P. Zhuang and F. Liu, "Implicit difference approximation for the time fractional diffusion equation," Journal of Applied Mathematics and Computing, vol. 22, no. 3, pp. 87-99, 2006.

[14] B. Jin, B. Li, and Z. Zhou, "An analysis of the Crank-Nicolson method for subdiffusion," IMA Journal of Numerical Analysis, vol. 38, no. 1, pp. 518-541, 2018.

[15] D. Li, W. Sun, and C. Wu, "A novel numerical approach to time-fractional parabolic equations with nonsmooth solutions," Numerical Mathematics: Theory, Methods and Applications, vol. 14, no. 2, pp. 355-376, 2021.

[16] L. Li and D. Li, "Exact solutions and numerical study of time fractional Burgers' equations," Applied Mathematics Letters, vol. 100, p. 106011, 2020.

[17] C. Li and W. Deng, "High order schemes for the tempered fractional diffusion equations," Advances in Computational Mathematics, vol. 42, no. 3, pp. 543-572, 2016.

[18] S. B. Yuste, "Weighted average finite difference methods for fractional diffusion equations," Journal of Computational Physics, vol. 216, no. 1, pp. 264-274, 2006.

[19] S. B. Yuste and L. Acedo, "An explicit finite difference method and a new von Neumann-type stability analysis for fractional diffusion equations," SIAM Journal on Numerical Analysis, vol. 42, no. 5, pp. 1862-1874, 2005.

[20] C. Çelik and I. Duman, "Crank-Nicolson method for the fractional diffusion equation with the Riesz fractional derivative," Journal of Computational Physics, vol. 231, no. 4, pp. 17431750, 2012.

[21] X. Lin and C. Xu, "Finite difference/spectral approximations for the time-fractional diffusion equation," Journal of Computational Physics, vol. 225, no. 2, pp. 1533-1552, 2007.

[22] X. Chen, Y. Di, J. Duan, and D. Li, "Linearized compact ADI schemes for nonlinear time-fractional Schrodinger equations," Applied Mathematics Letters, vol. 84, pp. 160-167, 2018.

[23] D. Li, J. Wang, and J. Zhang, "Unconditionally convergent \$L1\$-Galerkin FEMs for nonlinear time-fractional Schrödinger equations," SIAM Journal on Scientific Computing, vol. 39, no. 6, pp. A3067-A3088, 2017.

[24] Z. Sun, J. Zhang, and Z. Zhang, "Optimal error estimates in numerical solution of time fractional Schrödinger equations on unbounded domains," East Asian Journal on Applied Mathematics, vol. 8, no. 4, pp. 634-655, 2019.

[25] M. Gunzburger and J. Wang, "A second-order CrankNicolson method for time-fractional PDEs," International Journal of Numerical Analysis and Modeling, vol. 16, no. 2, pp. 225-239, 2019.

[26] N. H. Sweilam, H. Moharram, N. K. A. Abdel Moniem, and S. Ahmed, "A parallel Crank-Nicolson finite difference method for time-fractional parabolic equation," Journal of Numerical Mathematics, vol. 22, no. 4, pp. 363-382, 2014.

[27] N. H. Sweilam, M. M. Khader, and A. M. Mahdy, "Crank-Nicolson finite difference method for solving time-fractional diffusion equation," Journal of Fractional Calculus and Applications, vol. 2, no. 2, pp. 1-9, 2012. 
[28] Q. Zhang, M. Ran, and D. Xu, "Analysis of the compact difference scheme for the semilinear fractional partial differential equation with time delay," Applicable Analysis, vol. 96, no. 11, pp. 1867-1884, 2016.

[29] F. A. Rihan, "Computational methods for delay parabolic and time-fractional partial differential equations," Numerical Methods for Partial Differential Equations, vol. 26, no. 6, pp. 1556-1571, 2010.

[30] M. Li, C. Huang, and F. Jiang, "Galerkin finite element method for higher dimensional multi-term fractional diffusion equation on non-uniform meshes," Applicable Analysis, vol. 96, no. 8, pp. 1269-1284, 2016.

[31] J. Cao and C. Xu, "A high order schema for the numerical solution of the fractional ordinary differential equations," Journal of Computational Physics, vol. 238, pp. 154-168, 2013.

[32] M. Stynes, E. O'riordan, and J. L. Gracia, "Error analysis of a finite difference method on graded meshes for a timefractional diffusion equation," SIAM Journal on Numerical Analysis, vol. 55, no. 2, pp. 1057-1079, 2017.

[33] D. Li, H.-L. Liao, W. Sun, J. Wang, and J. Zhang, "Analysis of L1-Galerkin FEMs for time-fractional nonlinear parabolic problems," Communications in Computational Physics, vol. 24, pp. 86-103, 2018.

[34] D. Li, J. Zhang, and Z. Zhang, "Unconditionally optimal error estimates of a linearized Galerkin method for nonlinear time fractional reaction-subdiffusion equations," Journal of Scientific Computing, vol. 76, no. 2, pp. 848-866, 2018.

[35] R. Lin and F. Liu, "Fractional high order methods for the nonlinear fractional ordinary differential equation," Nonlinear Analysis: Theory Methods \& Applications, vol. 66, no. 4, pp. 856-869, 2007.

[36] Y. Liu, Y. Du, H. Li, S. He, and W. Gao, "Finite difference/finite element method for a nonlinear time-fractional fourth-order reaction-diffusion problem," Computers \& Mathematcs with Applications, vol. 70, no. 4, pp. 573-591, 2015.

[37] C. Li and F. Zeng, "Finite difference methods for fractional differential equations," International Journal of Bifurcation and Chaos, vol. 22, no. 4, p. 1230014, 2012.

[38] B. Jin, B. Li, and Z. Zhou, "Numerical analysis of nonlinear subdiffusion equations," SIAM Journal on Numerical Analysis, vol. 56, no. 1, pp. 1-23, 2018.

[39] H. Liao, D. Li, and J. Zhang, "Sharp error estimate of the nonuniform L1 formula for linear reaction-subdiffusion equations," SIAM Journal on Numerical Analysis, vol. 56, no. 2, pp. 1112-1133, 2018.

[40] D. Li, C. Wu, and Z. Zhang, "Linearized Galerkin FEMs for nonlinear time fractional parabolic problems with nonsmooth solutions in time direction," Journal of Scientific Computing, vol. 80, no. 1, pp. 403-419, 2019.

[41] Z. Wang and S. Vong, "Compact difference schemes for the modified anomalous fractional sub-diffusion equation and the fractional diffusion-wave equation," Journal of Computational Physics, vol. 277, pp. 1-15, 2014.

[42] F. Zeng, C. Li, F. Liu, and I. Turner, "Numerical algorithms for time-fractional subdiffusion equation with second-order accuracy," SIAM Journal on Scientific Computing, vol. 37, no. 1, pp. A55-A78, 2015.

[43] X. Zhao and Z. Sun, "Compact Crank-Nicolson schemes for a class of fractional Cattaneo equation in inhomogeneous medium," Journal of Scientific Computing, vol. 62, no. 3, pp. 747-771, 2015.
[44] C. Lubich, "Convolution quadrature and discretized operational calculus. I," Numerische Mathematik, vol. 52, no. 2, pp. 129-145, 1988.

[45] B. Jin, B. Li, and Z. Zhou, "Correction of high-order BDF convolution quadrature for fractional evolution equations," SIAM Journal on Scientific Computing, vol. 39, no. 6, pp. A3129A3152, 2017.

[46] N. Liu, Y. Liu, H. Li, and J. Wang, “Time second-order finite difference/finite element algorithm for nonlinear timefractional diffusion problem with fourth-order derivative term," Computers \& Mathematcs with Applications, vol. 75, no. 10, pp. 3521-3536, 2018.

[47] L. Li, B. Zhou, X. Chen, and Z. Wang, "Convergence and stability of compact finite difference method for nonlinear time fractional reaction-diffusion equations with delay," Applied Mathematics and Computation, vol. 337, pp. 144-152, 2018.

[48] A. S. Hendy, V. G. Pimenov, and J. E. Macías-Díaz, "Convergence and stability estimates in difference setting for timefractional parabolic equations with functional delay," Numerical Methods for Partial Differential Equations, vol. 36, no. 1, pp. 118-132, 2019.

[49] A. S. Hendy and J. E. Macías-Díaz, "A novel discrete Gronwall inequality in the analysis of difference schemes for timefractional multi-delayed diffusion equations," Communications in Nonlinear Science and Numerical Simulation, vol. 73, pp. 110-119, 2019.

[50] V. Thomée, Galerkin Finite Element Methods for Parabolic Problems, vol. 1054, Springer, 1984.

[51] D. Kumar, S. Chaudhary, and V. V. K. S. Kumar, "Fractional Crank-Nicolson-Galerkin finite element scheme for the time-fractional nonlinear diffusion equation," Numerical Methods for Partial Differential Equations, vol. 35, no. 6, pp. 2056-2075, 2019.

[52] R. Rannacher and R. Scott, "Some optimal error estimates for piecewise linear finite element approximations," Mathematics of Computation, vol. 38, no. 158, pp. 437-445, 1982. 